\title{
A Search for Axion-like Particles with a Horizontally Polarized Beam in a Storage Ring
}

\author{
Edward J. Stephenson ${ }^{1}$ \\ Indiana University Center for Spacetime Symmetries, Department of Physics \\ Bloomington, Indiana 47405 USA \\ E-mail: stephenelindiana.edu
}

On behalf of the JEDI Collaboration

\begin{abstract}
A new method has been demonstrated using a polarized deuteron beam in the storage ring COSY to search for axion-like particles. The signal would be a resonant jump in the vertical polarization component of a polarized beam whose spins are rotating in the ring plane under the influence of the COSY bending magnets. The axion would cause the electric dipole moment (EDM) of the beam particles to oscillate. If this frequency happens to coincide with the polarization rotation rate in the ring plane during a scan of the beam momentum, then the torques on the polarization induced through the EDM by the radial electric field in the beam frame will accumulate, leading to a change in the vertical polarization component. In the spring of 2019 , we used a $0.97-\mathrm{GeV} / \mathrm{c}$ vector-polarized deuteron beam to successfully demonstrate the procedure for the search. The phase of the oscillating EDM with respect to the rotation of the polarized beam is unknown. In order to be sensitive to both cosine and sine components of the oscillation, we prepared four beam bunches for the ring with different polarization directions. Starting with vertical polarization following injection into the ring, an RF solenoid operating on the $(1+\mathrm{G} \gamma)$ harmonic of the beam revolution frequency was used to rotate the polarization into the horizontal plane. This yielded a polarization pattern in which two of the bunches had polarizations that were nearly orthogonal. By looking separately for signals on both bunches, a signal would be found for any value of the axion phase. Beam polarizations were measured using the WASA Forward Detector. In order to improve the horizontal polarization lifetime, the beam was electron cooled as well as bunched. Once the orbit was established with minimal steering corrections, the ring sextupole magnets were adjusted to maximize the horizontal polarization lifetime. All scans were made with lifetimes in excess of $500 \mathrm{~s}$. The sensitivity to an axion was tested and calibrated using an RF Wien filter with a horizontal magnetic field to create vertical polarization jumps during a momentum scan of COSY. In a series of scans spanning a $1.2 \%$ change in the neighborhood of $121 \mathrm{kHz}$ spin tune frequency (associated with the axion mass), no signals were seen that did not fit the statistical distribution that arises from event counting data collection.
\end{abstract}

The 18th International Workshop on Polarized Sources, Targets, and Polarimetry, PSTP2019 23 -27 September, 2019

Knoxville, Tennessee

\section{${ }^{1}$ Speaker}




\section{Introduction}

In the 60 years since the first measurement of a vanishing electric dipole moment (EDM) on the neutron [1], the upper limit has been reduced more than 6 orders of magnitude [2] to $1.8 \times 10^{-26} \mathrm{e} \cdot \mathrm{cm}$. The absence of $\mathrm{CP}$ violation at this level has led to the suggestion [3] that there is a pseudo-scalar field associated with a new particle, called the axion, that may be responsible for $\mathrm{CP}$ conservation at this level. This new particle could also be a candidate to explain dark matter $[4,5]$. The axion's coupling to gluons induces an oscillating component [6,7] in the nucleon electric dipole moment (EDM) $d_{n}$ so that

$$
d_{n}(t)=d_{0}+d_{1} \cos \left(m_{a} t+\varphi_{a}\right)
$$

where $m_{a}$ is the axion mass. The axion phase $\varphi_{a}$ is included as a reminder that any detection apparatus that employs an oscillating electromagnetic field to reveal an axion signal must take into account the phase difference between the axion and the field oscillations.

The concept of using a storage ring to search for a static EDM $\left(d_{0}\right)$ depends upon using the torque it would experience in the particle-frame radial electric field to rotate the spin direction of a spin polarized beam, as shown in Fig. 1 [8].

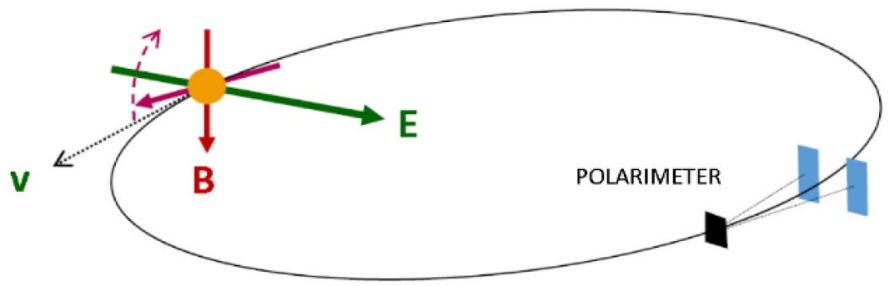

Figure 1: The polarized particle with spin initially along the momentum circulates in a storage ring whose electric and magnetic confining fields are chosen so that the in-plane precession rate of the polarization equals the rotation rate of the velocity, a condition known as "frozen spin." Under the influence of the torque generated by the E-field, the EDM and spin direction rotate out of the ring plane, creating a small vertical polarization component that appears as a left-right scattering asymmetry from the polarimeter target.

If the polarization is not frozen (is rotating in the ring plane at some frequency) and the charge alignment of the electric dipole is continually reversing at the same frequency, a resonance is created in which a similar rotation of the polarization out of the ring plane occurs. This makes possible a search for any axion in the neighborhood of the storage ring simply by putting the polarization initially in the ring plane and then ramping the machine revolution frequency while looking for a jump in the vertical polarization component. This paper reports the first successful test run of this concept using the COSY storage ring at the Forschungszentrum Jülich.

This search method assumes that the axion is coherent in space, causing $d_{l}$ for all beam particles to oscillate together no matter where they are along the ring. It assumes that axions are dense enough that whenever we look, an axion is there. And it assumes that the axion maintains coherence in time long enough for the resonance jump to be measured. For frequencies in the $\mathrm{kHz}$ to MHZ range explored in this way, these assumptions are likely met if the axions are bound to the local galaxy and their oscillations have a quality factor $>10^{6}$. Prior articles on this method have appeared in Refs. $[9,10]$.

\section{Machine Setup}

The demonstration run for the axion search used a $0.97 \mathrm{GeV} / \mathrm{c}$ vector polarized deuteron beam injected into the COSY storage ring [11]. The machine was filled with four bunches representing a single polarization state from the ion source. This state was alternated with an unpolarized state to complete the process cycle. The common practice of separate fills with opposite polarization states to suppress systematic errors was abandoned since the opposite fills would have different values of the axion phase $\varphi_{\mathrm{a}}$, giving a meaningless difference. At the same time if only a single bunch were present, random values of the phase might render any of the polarized beam fills insensitive to the axion if the EDM and longitudinal 
polarization components were oscillating out of phase. To circumvent this issue, the ring was operated on the fourth harmonic $(h=4)$ so that four deuteron beam pulses circulated simultaneously. The polarimeter was the WASA Forward Detector, which recorded a maximum asymmetry of $3 p_{y} A_{y} / 2=0.14$ with the polarized state enabled.

Figure 2 shows the polarimeter detector rate as a function of time within a single RF oscillation (or equivalently, a location on the ring circumference) and time in the store; the four beam bunches are prominent and well separated (left panel). When the rotation of the polarization from the vertical injection direction into the horizontal plane is accomplished by running an RF solenoid at the $f_{\mathrm{REV}}(1+G \gamma)=629771.9$ $\mathrm{Hz}$ harmonic ( $G$ is the deuteron anomaly and $f_{\mathrm{REV}}$ is the beam revolution frequency), the average distribution of the four polarization directions is shown by the right panel in Fig. 2.
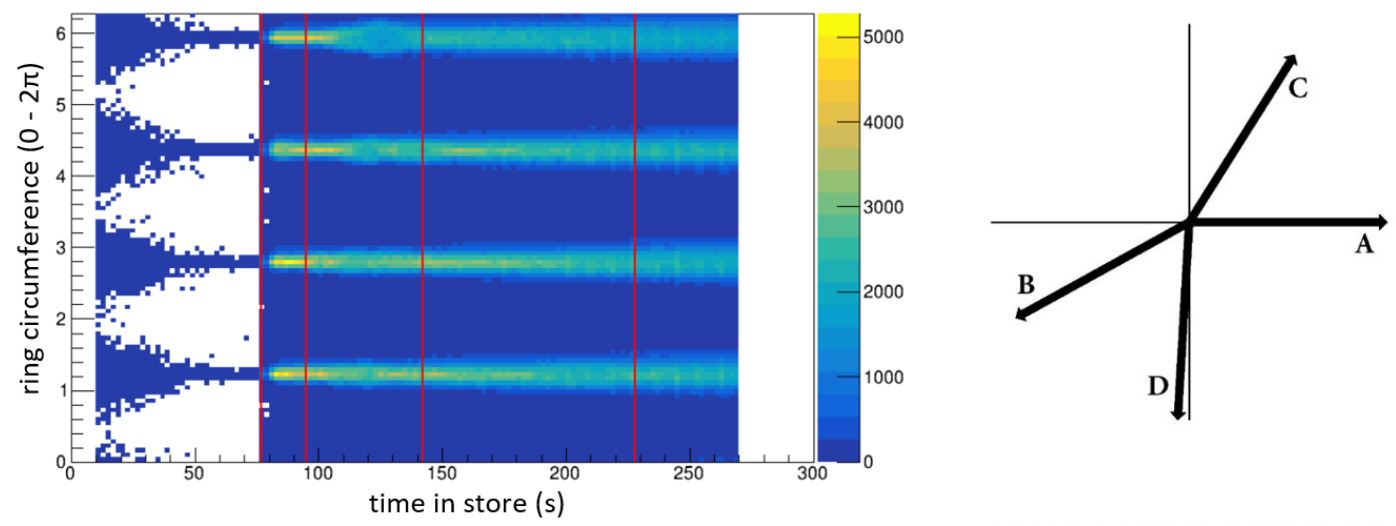

Figure 2: (left) Polarimeter count rate as a function of time within the store and time within one machine revolution (vertical axis in rad). (right) Average relative directions of the polarizations for the four beam bunches.

This distribution features two directions (A and D) that are nearly orthogonal, thus making sure that axions with any phase will be detected. This distribution also contains three pairs (AB, BC, and CD) of directions that are nearly opposite, thus offering a way to check for systematic effects in the measurement by comparing oppositely directed polarization jumps using a cross-ratio analysis [12]. Tests of the measurement of the horizontal polarization [13] produce relative polarization phases for the four bunches whose spacing confirms the pattern in the right panel of Fig. 2. The beam was electron cooled $(100 \mathrm{kV}$ maximum electron energy) and the ring sextupoles adjusted to ensure that the polarization lifetime was typically $500 \mathrm{~s}$ or greater [14].
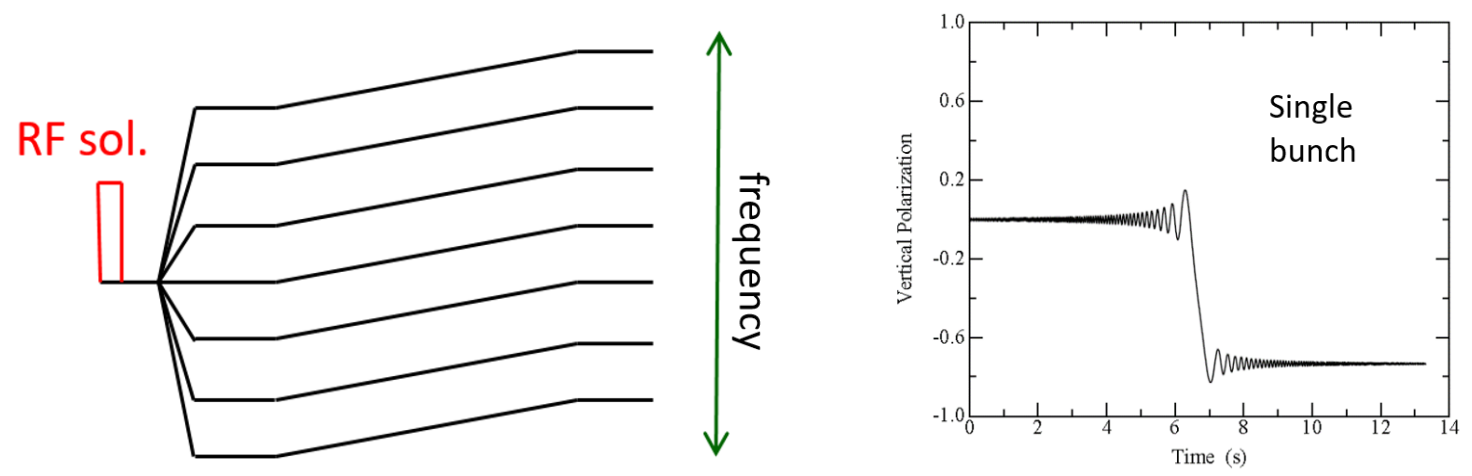

Figure 3: (left) Schematic diagram of the scanning process (see text). (right) A sample signal of a polarization jump from a model of the scanning process.

The scanning process is illustrated in Fig. 3 where the left panel shows the time evolution of the 
revolution frequency $f_{\text {REV }}$ (black curves) preceded by a time for the RF solenoid to produce a horizontally polarized beam (red). The scans overlap in frequency so as not to lose any axion signals. The RF solenoid requires that the beam be ramped to the resonant frequency, $f_{\mathrm{REV}}(1+G \gamma)$, needed for the rotating the polarization into the horizontal plane; once in place an additional fast ramp takes the beam to the starting point for each ramp. The right panel of Fig. 3 shows a model calculation of a polarization jump, which can be in either direction depending on the axion phase, for a single beam bunch.

\section{Calibration}

The sensitivity of the axion search may be determined in two ways. First, a spin tracking model that numerically integrated the rotations of the polarization turn by turn can be used to calculate the jump size for a given oscillating EDM $d_{l}$. Figure 4 shows the jump for phases of 0 (red) and $\pi / 2$ (magenta) as well as the total jump (black) as a function of $\varepsilon$, the ratio of the induced polarization rotation rate divided by the machine frequency. If a jump of a certain size is observed, this calibration connects that size to the size of the oscillating EDM shown as $2 \pi \varepsilon$ in radians.
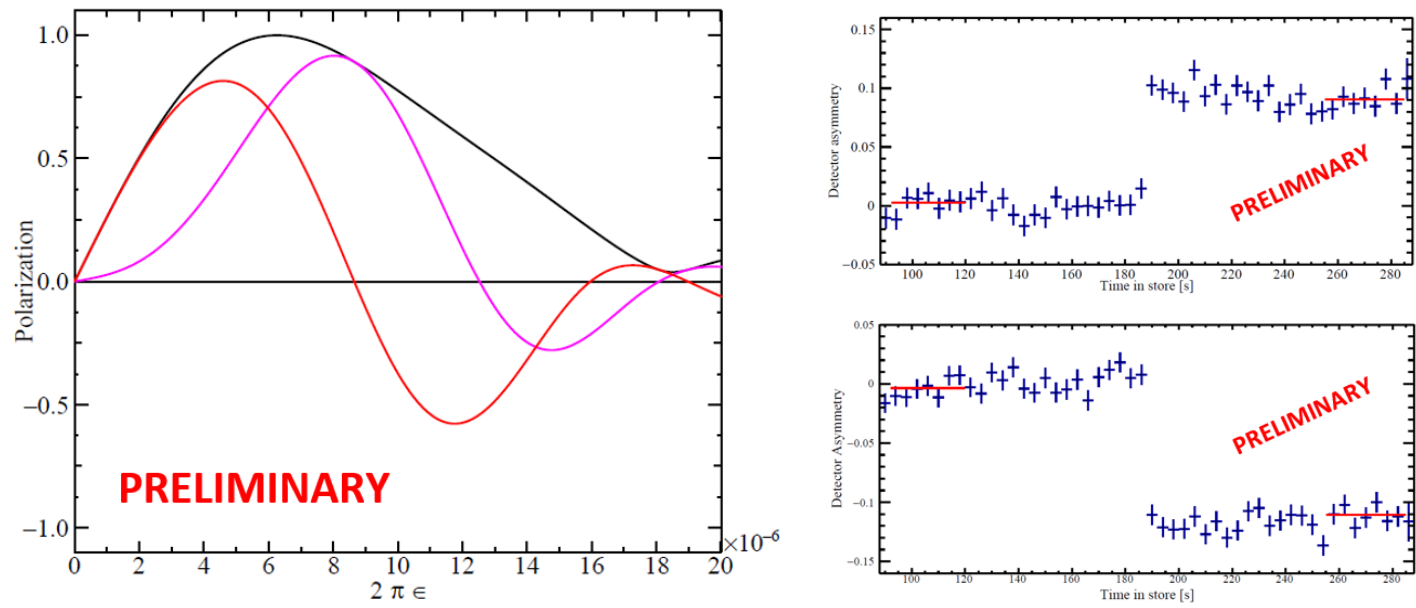

Figure 4: (left) Model polarization jump as a function of $\varepsilon$ (see text). (right) Jumps created by an RF Wien filter mounted to provide a horizontal oscillating B field.

By setting an RF Wien filter with sideways magnetic field to a fixed frequency, $f_{\mathrm{REV}}(1-G \gamma)$, and then scanning the machine revolution frequency across that resonance, a "test" polarization jump was created, as shown in the right panel of Fig. 4 for two fills from a series. As the phase between the machine and the RF Wien filter is random (like the axion phase), jumps varied in size and direction. These are among the largest. By modeling these jumps based on a calibration of driven polarization oscillations created from matching a stationary machine frequency to the Wien filter frequency, the formalism for handling such jumps may be confirmed.

\section{Results}

The oscillating EDM associated with the axion mass [6,7] experiences a torque from the radial electric field in the beam frame that closes the beam orbit in the storage ring. The beam polariation, and thus the EDM, also rotates with respect to this field at the spin tune frequency of $G \gamma f_{\mathrm{REV}}=120847 \mathrm{~Hz}$ just prior to any scans (left panel of Fig. 3). The 106 completed scans ranged from $850 \mathrm{~Hz}$ below the starting spin tune frequency to $612 \mathrm{~Hz}$ above the spin tune frequency, a total span of almost $1.2 \%$. The average asymmetry in the flat portions of the scan at either end (see left panel of Fig. 3) were compared and any differences larger than 2 standard deviations flagged during running for further consideration and possible repetition. For each setting of the scanning frequency range, at least 8 scans with polarized beam were completed. Jumps up to 5 standard deviations were recorded in single stores, but the distribution of jumps remained consistent with statistical expectations for no jump at all. The search found no candidates for an axion-like particle. The explored oscillating frequency region is indicated by a red mark in Fig. 5. 


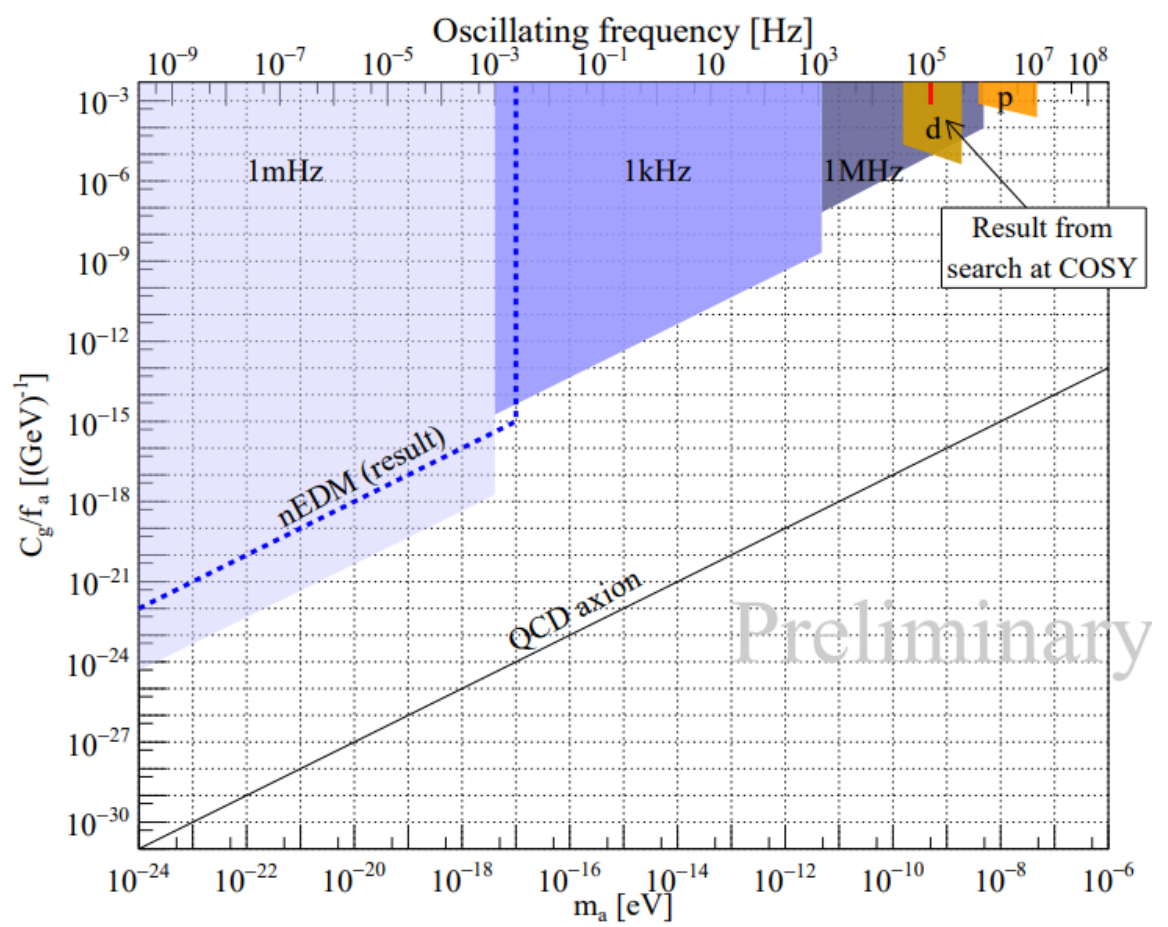

Figure 5: Location of present scan (red rectangle) in terms of the axion mass or frequency. The plot comes from Ref. [10] and shows areas of potential interest in terms of $C_{\mathrm{g}} / f_{\mathrm{a}}$, the ratio of the axion gluon coupling to the axion decay constant. Possible scans with the COSY storage ring are noted in yellow for protons and orange for deuterons. In the case of a suggested prototype ring (with both electric and magnetic fields for EDM ring development) three regions in blue indicate potential search areas. A blue dashed line marks a limit reported by a neutron EDM experiment [2].

\section{Conclusions}

An experiment at the COSY cooler synchrotron has demonstrated the possibility to search for axion-like particles by scanning for a resonance between the axion oscillating EDM and the rotation of an in-plane polarized beam stored in the ring. The signal is a jump in the vertical polarization as the resonance is crossed. By using four beams stored simultaneously, the search becomes independent of the relative phase between the axion and the rotating polarization. Sensitivity was demonstrated using a sideways oscillating magnetic field from an RF Wien filter to mimic the effects of an axion. The frequency range scanned was about $1.2 \%$ in frequency in the neighborhood of $121 \mathrm{kHz}$.

\section{References}

[1] J. H. Smith, E. M. Purcell, and N. F. Ramsey, "Experimental limit to the electric dipole moment of the neutron," Phys. Rev. 108, 120 (1957).

[2] C. Abel et al., "Measurement of the Permanent Electric Dipole Moment of the Neutron," Phys. Rev. Lett. 124, 081803 (2020).

[3] R. D. Peccei and H. Quinn, “CP Conservation in the Presence of Pseudoparticles," Phys. Rev. Lett. 38, 1440 (1977).

[4] I. G. Irastorza and J. Redondo, "New experimental approaches in the search for axion-like particles," Prog. Part. Nucl. Phys. 102, 89 (2018).

[5] P. W. Graham, I. G. Irastorza, S. K. Lamoreaux, A. Lindner, and K. A. Van Bibber, "Experimental Searches for the Axion and Axion-like Particles," Ann. Rev. Nucl. Part. Sci. 65, 485 (2015).

[6] P. W. Graham and S. Rajendran, "Axion dark matter detection with cold molecules," Phys. Rev. D 
84, 055013 (2011).

[7] P. W. Graham and S. Rajendran, "New observables for direct detection of axion dark matter," Phys. Rev. D 88, 035023 (2013).

[8] V. Anastassopoulas et al., "A storage ring experiment to detect a proton electric dipole moment," Rev. Sci. Instrum. 87, 115116 (2016).

[9] S. P. Chang et al., "Axionlike dark matter search using the storage ring EDM method," Phys. Rev. D 99, 083002 (2019).

[10] J. Pretz et al., "Influence of electron cooling on the polarization lifetime of a horizontally polarized storage ring beam," arXiv 1908.09678, to be published in EPJ.

[11] R. Maier, "Cooler synchrotron COSY - Performance and perspectives," Nucl. Instrum. Methods Phys. Res. A 390, 1 (1997).

[12] G. G. Ohlsen and P. W. Keaton, Jr., "Techniques for measurement of spin-1/2 and spin-1 polarization analyzing tensors," Nucl. Instrum. Methods 109, 41 (1973).

[13]Z. Bagdasarian et al., "Measuring the polarization of a rapidly precessing deuteron beam," Phys. Rev. ST Accel. Beams 17, 052803 (2014).

[14] G. Guidoboni et al. "How to Reach a Thousand-Second in-Plane Polarization Lifetime with 0.97$\mathrm{GeV} / \mathrm{c}$ Deuterons in a Storage Ring, Phys. Rev. Lett. 117, 054801 (2016). 
0
0
2
$\ddots$
$\tau$
1
0
0
0
0
0
0 